\title{
Veszprém Optimization Conference: Advanced Algorithms (VOCAL) 2010
}

\author{
Botond Bertók · Zoltán Süle · Tamás Terlaky
}

Published online: 8 November 2013

(C) Springer Science+Business Media New York 2013

The Faculty of Information Technology and the Veszprém Regional Committee of the Hungarian Academy of Sciences (MTA-VEAB) jointly organize the Veszprém Optimization Conference: Advanced Algorithms (VOCAL) conference series.

The VOCAL 2010 conference highlighted notable advances in the broadly defined area of optimization algorithms: continuous and discrete optimization, complexity, convergence properties, high-performance computational optimization, and important applications. VOCAL is a medium-scale event that provides an opportunity to review the state-of-the-art of optimization algorithms and their applications and to discuss emerging challenges and trends. The organizers aimed to facilitate interaction between young researchers and leading experts from the theoretical and applied optimization communities. During the three days of the conference, internationally renowned researchers from 20 countries on 4 continents delivered over 70 presentations reviewing their latest achievements.

We wish to express our sincere gratitude to the International Scientific Committee. Its members were: Rainer E. Burkard, Christodoulos A. Floudas, Katya Scheinberg, Fabio Schoen, Philippe Toint, Ya-xiang Yuan, and Tamás Terlaky. We also thank

B. Bertók · Z. Süle

Department of Systems and Computing Sciences, College of Technical Informatics, Pannon University, Egyetem u. 10, Veszprém 8200, Hungary

B. Bertók

e-mail: bertok@dcs.uni-pannon.hu

Z. Süle

e-mail: sule@dcs.uni-pannon.hu

T. Terlaky (凶)

Department of Industrial and Systems Engineering, Lehigh University, 200 West Packer Avenue,

Bethlehem, PA 18015-1582, USA

e-mail: terlaky@lehigh.edu 
the Organizing Committee for arranging a high-quality conference. This committee was chaired by Ferenc Friedler and included Botond Bertók, Tibor Csendes, Csaba Fábián, István Győri, Zoltán Horváth, Tibor Illés, Sándor Komlósi, István Maros, Zsolt Páles, Tamás Szántai, László Szeidl, and József Temesi.

This special issue presents some of the highlights of the conference, and we now briefly introduce each paper.

Szántai and Kovács introduce a special kind of $k-1$-width junction tree in order to approximate a joint probability distribution. They show that the best approximating $k-1$-width junction-tree probability distribution can be embedded into a $k$ th-order cherry-tree probability distribution. The authors present a greedy algorithm that gives good approximations in a reasonable computing time.

Mészaros presents a variant of the nested dissection algorithm incorporating special techniques that are beneficial for the graph partitioning problems arising in the ordering step of implementations of interior point methods. He discusses the behavior of the algorithm, presents numerical results, and gives comparisons with other sparse matrix-ordering algorithms.

Koltai and Tatay provide a general framework for assembly line balancing models with workforce skill constraints. Workers who are able to perform only simple tasks must be assigned low-skill tasks. High-skill tasks require higher than average skills. Finally, exclusive-skill tasks are performed by groups of workers who specialize in certain tasks. The authors summarize the mathematical description of the different skill constraints and show how simple assembly-line balancing models can take skills into account.

Király et al. define a Taguchi-type loss function to aggregate process constraints related to quality or safety requirements, target values, and desired product-quality ranges. They evaluate the loss function by Monte-Carlo simulation because the process is stochastic and use the gradient-free mesh adaptive direct search (MADS) algorithm to optimize the resulting robust cost function. The concept is illustrated by a well-known benchmark problem related to the control of a linear dynamical system and the model predictive control of a more complex nonlinear polymerization process.

Adonyi et al. develop a model based on a $P$-graph for a maintenance scheduling problem. It takes both day and night into account because buses often have some unused time during the day. Using the proposed method, a better maintenance schedule is developed where certain repair tasks occur between the morning and afternoon rush hours. This approach can reduce costs because repairs at night are more expensive.

Divéki and Imreh study two-dimensional clustering with the $l_{\infty}$ norm, so the clusters are actually squares. The goal is to minimize the total cost of the clusters used. They present a 7-competitive and an approximately 5.22-competitive algorithm and also give bounds on the possible ratios.

Veszprém, 10 October, 2013

Botond Bertók, Zoltán Süle, and Tamás Terlaky 